REVISTA MATEMÁTICA COMPLUTENSE

Volumen 11, número 1: 1998

http://dx.doi.org/10.5209/rev_REMA.1998.v11.n1.17325

\title{
Some remarks on the regularity of weak solutions of degenerate elliptic systems.
}

\author{
Luca ESPOSITO and Giuseppe MINGIONE
}

\begin{abstract}
We prove the existence of second derivatives of the weak solutions $u \in W^{1, p}$ of the degenerate system $\operatorname{div} A(D u)=0$, where no differentiability is supposed on the monotone vector field $A$ : $R^{n N} \longrightarrow R^{n N}$. We also give a boundedness result for the scalar case.
\end{abstract}

\section{Introduction}

The main result of this paper is the higher differentiability of local solutions of elliptic systems of the type

$$
\operatorname{div} A(D v)=0 \quad \text { in } \mathcal{D}^{\prime}(\Omega)
$$

where $\Omega$ is an open subset of $R^{n}$ and $A$ is a mapping from $R^{n N}$ into $R^{n N}$. This problem has been studied by several authors; we only quote $[\mathrm{AF}],[\mathrm{DB}], \mid \mathrm{Gi}],[\mathrm{GM}],[\mathrm{U}]$, where the reader can find further references. 'To get such regularity, it is necessary to impose a suitable ellipticity assumption on $A$. We want to recall that if $A$ is $C^{1}$ and satisfies the ellipticity condition

1991 Mathernatics Subject Classification: 35J60, 35D10.

Servicio Publicaciones Univ. Complutense. Madrid, 1998.

This work has been performed as a part of a National Research Project

supported by MURST (40\%). 


$$
\frac{\partial A_{\alpha}^{i}}{\partial z_{\beta}^{j}} \xi_{i}^{\alpha} \xi_{j}^{\beta} \geq \nu\left(\mu^{2}+|z|^{2}\right)^{\frac{p-2}{2}}|\xi|^{2}
$$

and the growth conditions

$$
\begin{gathered}
|A(z)| \leq L\left(\mu^{2}+|z|^{2}\right)^{\frac{p-1}{2}} \\
\left|\frac{\partial A_{\alpha}^{i}}{\partial z_{\beta}^{j}}(z)\right| \leq \Lambda\left(\mu^{2}+|z|^{2}\right)^{\frac{p-2}{2}}
\end{gathered}
$$

for $\nu, L, \Lambda>0, p>1$, (see [Gi]), then, denoting by $u$ a solution of (1), one can prove that, if $\mu>0$ then

$$
u \in W_{l o c}^{2,2}(\Omega)
$$

and if, $0 \leq \mu$ then:

$$
V(D u)=\left(\mu^{2}+|D u|^{2}\right)^{\frac{p-2}{4}} D u \in W_{l o c}^{1,2}
$$

However in some recent papers (see $[\mathrm{E}],[\mathrm{EFL}],[\mathrm{FF}]$ ) it has been observed, in the case that (1) is the Euler-Lagrange system of a variational integral, that condition (4) can be dropped while the condition (2) can be replaced by a weaker form of ellipticity. In this paper we prove, in the general framework of the systems, the existence of second derivatives of the local solutions of (1), assuming that $A: R^{n N} \rightarrow R^{n N}$ is a continuous map satisfying (3) and the monotonicity condition

$$
\left\langle A\left(z_{1}\right)-A\left(z_{2}\right), z_{1}-z_{2}\right\rangle \geq \nu\left(\mu^{2}+\left|z_{1}\right|^{2}+\left|z_{2}\right|^{2}\right)^{\frac{p-2}{2}}\left|z_{1}-z_{2}\right|^{2}
$$

We distinguish the scalar case from the vectorial case, in fact in the scalar case $(N=1)$ we are also able to prove the local boundedness of the gradient of the local solution of (1). The proof of this results rests on the observation that the classical integral estimate involving the second derivatives of the weak solutions of a nondegenerate elliptic system satisfying (2)-(4) (see for example [Gi], chapter 8):

$$
\int_{B_{\rho}}\left(\mu^{2}+|D u|^{2}\right)^{\frac{p-2}{2}}\left|D^{2} u\right|^{2} d x \leq \frac{C}{(R-\rho)^{2}} \int_{B_{R}}\left(\mu^{2}+|D u|^{2}\right)^{\frac{p}{2}} d x
$$


can be proved with the constant $C$ not depending on $\Lambda$ and $\mu$ (see propositions 1 and 2, below).

Then we show (see lemma 2) that it is possible to find an approximation of the nondifferentiable, degenerate vector field $A$, by a sequence of smooth vector fields $A_{n}$ satisfying conditions (2)-(4) for different constants $\mu$. Finally the result follows by a simple approximation argument based on the monotonicity assumption made on $A$ and the stability of the constats in the last inequality mentioned before.

\section{Preliminary results and lemmas}

In the following $\Omega$ denotes a bounded open set in $R^{n}, B_{R}\left(x_{o}\right)$ will indicate the ball with centre in $x_{o}$ and radius $R$. Moreover $\mathrm{c}$ will denote a generic constsant, wich may vary throught the paper. If $u$ is integrable in $B_{R}\left(x_{o}\right)$ we set:

$$
(u)_{x_{0, R}}=\frac{1}{\omega_{n} R^{n}} \int_{B_{R}\left(x_{o}\right)} u d x=f_{B_{R}\left(x_{o}\right)} u d x .
$$

We will simply write $B_{R}$ in place of $B_{R}\left(x_{o}\right)$ when no confusion will arise. Let $A: R^{n N} \rightarrow R^{n N}$ be a continuous function. We shall deal with the equation

$$
\operatorname{div} A(D v)=0 \quad \text { in } \mathcal{D}^{\prime}(\Omega)
$$

where $v \in W^{1, p}(\Omega), \quad p>1$, and $N \geq 1$.

We will assume that $A(z)$ grows polinomially like $|z|^{p-1}$ and satisfies a monotonicity assumption, namely that

$$
\begin{gathered}
|A(z)| \leq L\left(\mu^{2}+|z|^{2}\right)^{\frac{p-1}{2}} \\
\left\langle A\left(z_{1}\right)-A\left(z_{2}\right), z_{1}-z_{2}\right\rangle \geq \nu\left(\mu^{2}+\left|z_{1}\right|^{2}+\left|z_{2}\right|^{2}\right)^{\frac{p-2}{2}}\left|z_{1}-z_{2}\right|^{2}
\end{gathered}
$$

for some $\mu \geq 0, L>0$ and for every $z \in R^{n N}$. Without loss of generality we will suppose that $\mu \leq 1$. We say that $u \in W^{1, p}\left(\Omega, R^{N}\right)$ is a weak solution of (1) if and only if

$$
\int_{\Omega} A_{\alpha}^{i}(D u) D_{\alpha} \varphi=0 \quad \forall \varphi \in W_{o}^{1, p}(\Omega)
$$


for $\alpha=1, \ldots . n ; i=1, \ldots . N$. Now we estabilish two Propositions that are crucial in the approximation argument used in the proof of the main result.

Vectorial case.

Proposition 1. Let $A: R^{n N} \rightarrow R^{n N}$ be a $C^{1}$ function satisfying (7), and assume that there exist $\nu, \Lambda>0$ such that for any $z, \xi \in R^{n N}$

$$
\begin{gathered}
\frac{\partial A_{\alpha}^{i}}{\partial z_{\beta}^{j}}(z) \xi_{i}^{\alpha} \xi_{j}^{\beta} \geq \nu\left(\mu^{2}+|z|^{2}\right)^{\frac{p-2}{2}}|\xi|^{2} \\
\left|\frac{\partial A_{\alpha}^{i}}{\partial z_{\beta}^{j}}(z)\right| \leq \Lambda\left(\mu^{2}+|z|^{2}\right)^{\frac{p-2}{2}}
\end{gathered}
$$

where $\mu>0$. If $u \in W^{1,2}\left(\Omega, R^{N}\right)$ is a weak solution of (6) in $\Omega$ and if $p \geq 2$ then:

$$
u \in W_{l \infty c}^{2,2}(\Omega)
$$

while if $1<p<2$ then

$$
u \in W_{l o c}^{2, p}(\Omega) .
$$

Moreover there exists a constant $C$ depending on $n, N, p, L, \nu$ but not on $\Lambda, \mu$ or $u$ such that if $B_{R}\left(x_{o}\right) \subset \Omega, 0<\rho<R$ :

$$
\int_{B_{\rho}}\left(\mu^{2}+|D u|^{2}\right)^{\frac{p-2}{2}}\left|D^{2} u\right|^{2} d x \leq \frac{C}{(R-\rho)^{2}} \int_{B_{R}}\left(\mu^{2}+|D u|^{2}\right)^{\frac{p}{2}}
$$

Remark 1. We want to stress that the constant $C$ appearing in (12) does not depend on $\Lambda, \mu, u$. Actually, under assumption of Proposition 1 it is well known that local solutions $u$ of (1) have second derivatives satisfying (12), (see [Gi]), nevertheless it is not obvious that estimate (12) holds with the nice dependence quoted above. Now we state a technical lemma that we need for the proof of Proposition 1.

Lemma 1. Under the assumption of Proposition 1 we have

$$
\int_{\Omega} A_{\alpha}^{i}(D u) D_{\alpha} \varphi=0 \quad i=1, \ldots ., N
$$

for any $\varphi \in W^{1,1}\left(\Omega, R^{N}\right)$ with compact support in $\Omega$ and such that

$$
A_{\alpha}^{i}(D u) \varphi, \quad D_{\alpha}\left(A_{\alpha}^{i}(D u)\right) \varphi^{i}, \quad A_{\alpha}^{i}(D u) D_{\alpha} \varphi^{i},
$$


are in $L^{1}(\Omega)$ for every $i=1, \ldots ., N ; \alpha=1, \ldots \ldots, n$.

Proof. Follows from [EFL] Lemma 2.

\section{Proof of Proposition 1.}

Let us fix $B_{R}\left(x_{o}\right) \subset \Omega, 0<\rho<R$ and $\eta \in C_{o}^{2}\left(B_{R}\right), 0 \leq \eta \leq 1, \eta \equiv 1$ on $B_{\rho},|D \eta|^{2}+\left|D^{2} \eta\right| \leq c /(R-r)^{2}$. Fix also $M>0$ and $\psi \in C^{1}(R)$ such that $0 \leq \psi \leq 1, \psi=1$ if $t<\frac{M}{2}, \psi(t)=0$ if $t \geq M$ and $\mid \psi^{\prime}(t) \leq \frac{4}{M}$. Let us define

$$
\varphi(x)=\triangle_{-h, s}\left[\eta^{2}(x) D_{s} u(x) \psi\left(|D u(x)|+\left|D u\left(x+h e_{s}\right)\right|\right)\right],
$$

where we denote, for $g \in L_{\text {loc }}^{1}\left(R^{n}\right)$ and $s \in\{1, \ldots, n\}$, by $\Delta_{h, s} g$ (or simply $\Delta_{h} g$ if no confusion arises) the difference quotient

$$
\triangle_{h, s} g(x)=\frac{g\left(x+h e_{s}\right)-g(x)}{h}
$$

where $e_{s}$ is the unit vector in the s-direction, $h \in R$. If $|h|$ is sufficently small, the function $\varphi(x)$ has compact support in $B_{R}$. It is easy to check that conditions (14) stated in Lemma 1 are satisfied by $\varphi$ because $u \in W_{l o c}^{2,1}$ and by a standard difference quotient argument $A_{\alpha}^{i}(D u) \in$ $W_{l o c}^{1,1}\left(\Omega, R^{N}\right)$, moreover $\varphi=0$ whenever $|D u(x)| \geq M$. Therefore we get from (13)

$$
\begin{aligned}
& \int_{\Omega} \triangle_{h}\left(A_{\alpha}^{i}(D u)\right) \eta^{2} D_{\alpha s} u^{i} \psi\left(|D u(x)|+\left|D u\left(x+h e_{s}\right)\right|\right) d x \\
& =-\int_{\Omega} \triangle_{h}\left(A_{\alpha}^{i}(D u)\right) \eta^{2} D_{s} u^{i} \psi^{\prime}\left(|D u(x)|+\left|D u\left(x+h e_{s}\right)\right|\right) . \\
& \quad D_{\alpha}\left(|D u(x)|+\left|D u\left(x+h e_{s}\right)\right|\right) d x \\
& +2 \int_{\Omega} A_{\alpha}^{i}(D u) \Delta_{-h}\left[\eta D_{\alpha} \eta D_{s} u^{i} \psi\left(|D u(x)|+\left|D u\left(x+h e_{s}\right)\right|\right)\right] d x .
\end{aligned}
$$

Now we recall that under the assumption of Proposition $1 u \in W_{l o c}^{2,2}$ if $p \geq 2$, while $A_{\alpha}^{i}(D u) \in W_{l o c}^{1,2}$ and $D^{2} u 1_{\{|D u| \leq M\}}$ is in $L_{l o c}^{2}$ (see lemma 2.5 in [AF]), then we pdss to the limit in the last formula to get:

$$
\int_{\Omega} \frac{\partial A_{\alpha}^{i}}{\partial z_{\beta}^{j}}(D u) \eta^{2} D_{\alpha s} u^{i} D_{\beta s} u^{j} \psi(2|D u(x)|) d x
$$




$$
\begin{aligned}
= & -2 \int_{\Omega} \frac{\partial A_{\alpha}^{i}}{\partial z_{\beta}^{j}}(D u) \eta^{2} D_{s} u^{i} D_{\beta s} u^{j} \psi^{\prime}(2|D u(x)|) D_{\alpha}(|D u(x)|) d x \\
& +2 \int_{\Omega} A_{\alpha}^{i}(D u) D_{\alpha}\left(\eta D_{\alpha} \eta D_{s} u^{i} \psi(2|D u(x)|)\right) d x .
\end{aligned}
$$

Now from $(7),(10),(11)$ we get, summing up with respect to $s$ :

$$
\begin{aligned}
& \int_{\Omega}\left(\mu^{2}+|D u|^{2}\right)^{\frac{p-2}{2}}\left|D^{2} u\right|^{2} \eta^{2} \psi(2|D u(x)|) d x \\
& \leq c \Lambda \int_{\Omega}\left(\mu^{2}+|D u|^{2}\right)^{\frac{p-2}{2}}\left|D^{2} u\right|^{2} \eta^{2}|D u| \psi^{\prime}(2|D u(x)|) d x \\
& +c \int_{\Omega}\left(\mu^{2}+|D u|^{2}\right)^{\frac{p-1}{2}}\left|D_{\alpha}\left(\eta D_{\alpha} \eta D u \psi(2|D u(x)|)\right)\right| d x
\end{aligned}
$$

where $c$ does not depend on $\Lambda$ or $\mu$. Since $\left|\psi^{\prime}\right| \leq \frac{4}{M} 1_{[M / 2, M]}$, using Young inequality we obtain

$$
\begin{aligned}
& \int_{\Omega}\left(\mu^{2}+|D u|^{2}\right)^{\frac{p-2}{2}}\left|D^{2} u\right|^{2} \eta^{2} \psi(2|D u(x)|) d x \\
& \leq c(\Lambda+1) \int_{\{M / 2 \leq|D u(x)| \leq M\}}\left(\mu^{2}+|D u|^{2}\right)^{\frac{p-2}{2}} \\
& \quad\left|D^{2} u\right|^{2} \eta^{2}|D u| \psi^{\prime}(2|D u(x)|) d x \\
& +\int_{\Omega}\left(\mu^{2}+|D u|^{2}\right)^{\frac{p}{2}}\left(|D \eta|^{2}+\left|D^{2} \eta\right|\right) \psi(2|D u(x)|) d x .
\end{aligned}
$$

From this inequality, letting $M \longrightarrow \infty$ (12) follows.

\section{Scalar case.}

Proposition 2. Let $A: R^{n} \rightarrow R^{n}$ be a $C^{1}$ function satisfying (7), and assume that there exist $\mu, \Lambda>0$ such that for any $z, \xi \in R^{n}$

$$
\begin{gathered}
\frac{\partial A_{\alpha}}{\partial z_{\beta}}(z) \xi^{\alpha} \xi^{\beta} \geq \nu\left(\mu^{2}+|z|^{2}\right)^{\frac{p-2}{2}}|\xi|^{2} \\
\left|\frac{\partial A_{\alpha}}{\partial z_{\beta}}(z)\right| \leq \Lambda\left(\mu^{2}+|z|^{2}\right)^{\frac{p-2}{2}}
\end{gathered}
$$

where $\mu>0$. If $u \in W^{1, p}(\Omega)$ is a local solution of (1) in $\Omega$ then $u \in$ $W_{\text {loc }}^{2,2}(\Omega) \cap W_{\text {loc }}^{1, \infty}(\Omega)$. Moreover there exists a constant $C$ depending on 
$n, p, L, \nu$ but not on $\Lambda, \mu$ or $u$ such that if $B_{R}\left(x_{0}\right) \subset \Omega, 0<\rho<R$, the following estimates hold

$$
\begin{gathered}
\int_{B_{\rho}}\left(\mu^{2}+|D u|^{2}\right)^{\frac{p-2}{2}}\left|D^{2} u\right|^{2} d x \leq \frac{C}{(R-\rho)^{2}} \int_{B_{R}}\left(\mu^{2}+|D u|^{2}\right)^{\frac{p}{2}} d x \\
\sup _{B_{\rho}}|D u|^{p} \leq c(\rho) C f_{B_{R}}\left(\mu^{2}+|D u|^{2}\right)^{\frac{p}{2}} d x .
\end{gathered}
$$

Remark 2. The proposition above is the counterpart of Proposition 1 in the scalar case, and again the main point is the independence of (18),(19) of $\Lambda, \mu, u$. Moreover we observe that in this case, under the hypothesis of Proposition 2 it is known that $D u \in L_{\text {loc }}^{\infty}(\Omega)$, and this implies that $u \in W_{l o c}^{2,2}(\Omega)$ for any $1<p<\infty$.

\section{Proof of Proposition 2.}

The proof of (18) may be simplified in this case, because we know that $D u \in L_{l o c}^{\infty}\left(\Omega, R^{n}\right)$ and so we can just take $\varphi=\Delta_{-h, 9}\left(\eta^{2} D_{s} u\right)$ instead of (15). To prove (19) choose $\varphi=D_{s}\left(\eta^{2} H^{\beta} D_{s} u\right)$ in (9), where

$$
H(D u)=\mu^{2}+|D u|^{2}
$$

$s=1, \ldots, n, \eta \in C_{o}^{1}\left(B_{R}\right), 0 \leq \eta \leq 1, \eta \equiv 1$ on $B_{\rho}$ and $|D \eta|^{2}+\left|D^{2} \eta\right| \leq$ $\frac{c}{(R-\rho)^{2}}$. This is an admissible test function because $u \in W_{l o c}^{2,2}(\Omega) \cap$ $W_{l o c}^{1, \infty}(\Omega)$, then we get

$$
\int_{B_{R}} A_{\alpha}(D u) D_{s}\left(D_{\alpha}\left(H^{\beta} D_{s} u\right)\right) \eta^{2} d x=-2 \int_{B_{R}} \eta A_{\alpha}(D u) D_{s}\left(H^{\beta} D_{s} u\right) D_{\alpha} \eta
$$

Integrating by part the first integral, we have

$$
\begin{aligned}
& \int_{B_{R}} \frac{\partial A_{\alpha}(D u)}{\partial z_{\beta}} D_{s \beta} u D_{\alpha}\left(H^{\beta} D_{s} u\right) \eta^{2} d x \\
& =2 \int_{B_{R}} \eta A_{\alpha}(D u) D_{s}\left(H^{\beta} D_{s} u\right) D_{\alpha} \eta d x \\
& -2 \int_{B_{R}} \eta A_{\alpha}(D u) D_{\alpha}\left(H^{\beta} D_{s} u\right) D_{s} \eta d x,
\end{aligned}
$$


then using (7) we get

$$
\begin{aligned}
& \int_{B_{R}} \frac{\frac{\partial A_{\alpha}(D u)}{\partial z_{\beta}} D_{s \beta} u D_{s \alpha} u H^{\beta} \eta^{2}}{d x} \\
& +\beta \int_{B_{R}} \frac{\frac{\partial A_{\alpha}(D u)}{\partial z_{\beta}} D_{s \beta} u D_{s} u D_{\alpha}\left(|D u|^{2}\right) H^{\beta-1} \eta^{2} d x}{\leq c\left(n, p_{,} L\right) \int_{B_{R}} H^{\frac{p-1}{2}} \eta|D \eta|\left[H^{\beta}\left|D^{2} u\right|+\beta H^{\beta-1}|D u| D\left(|D u|^{2}\right)\right] d x .}
\end{aligned}
$$

Now we use (16) to obtain, summing up on $s$

$$
\begin{aligned}
& \nu \int_{B_{R}} H^{\beta+\frac{p-2}{2}}\left|D^{2} u\right|^{2} \eta^{2} d x+\frac{\nu \beta}{2} \int_{B_{A}} H^{\beta-1+\frac{p-2}{2}}\left|D\left(|D u|^{2}\right)\right|^{2} \eta^{2} d x \\
& \leq c(n, p, L) \int_{B_{R}} H^{\beta+\frac{p-1}{2}} \eta\left|D \eta \| D^{2} u\right| d x \\
& +c(n, p, L) \beta \int_{B_{\text {A }}} H^{\beta-\frac{1}{2}+\frac{p-1}{2}}\left|D\left(|D u|^{2}\right) \| D \eta\right| \eta d x .
\end{aligned}
$$

Now we observe that

$$
\int_{B_{\lambda L}} H^{\beta-1+\frac{p-2}{2}}\left|D\left(|D u|^{2}\right)\right|^{2} \eta^{2} d x \leq c(n) \int_{B_{n}} H^{\beta+\frac{p-2}{2}}\left|D^{2} u\right|^{2} \eta^{2} d x
$$

and apply Young inequality to get

$$
\int_{B_{R}} H^{\beta-1+\frac{p-2}{2}}\left|D\left(|D u|^{2}\right)\right|^{2} \eta^{2} d x \leq \underline{\frac{c(n, p, L)}{\nu^{2}}} \int_{B_{R}} H^{\beta+\frac{p}{2}}|D \eta|^{2} d x .
$$

Setting $\gamma=\frac{p}{4}+\frac{\beta}{2} \geq \frac{p}{4} ;$ the above inequality implies

$$
\int_{B_{R}}\left|D\left(H^{\gamma} \eta\right)\right|^{2} \cdot d x \leq c(n, p, L, \nu) \gamma^{2} \int_{B_{R}} H^{2 \gamma}|D \eta|^{2} d x .
$$

Using now Poincaré inequality we deduce

$$
\left\|H^{\gamma} \eta\right\|_{L^{2 \times}\left(B_{R}\right)} \leq c(n, p, L, \nu) \gamma\left\|H^{\gamma} D \eta\right\|_{L^{2}\left(B_{1}\right)},
$$

where $\chi=\frac{N}{N-2}$ if $N \geq 3$, or any number grather then 1 if $N=2$. From this inequality by a straightforward application of Moser's technique we get (19). 
Now we state a simple approximation lemma that will allow us to manage the case when upper estimates on $|D A|$ are not available and $\mu=0$.

Lemma 2. Let $A: R^{n N} \rightarrow R^{n N}$ be a continuous function satisfying (7) and $(8)$ where $\mu \geq 0$. Then there exists a sequence of $C^{1}\left(R^{n N}, R^{n N}\right)$ functions $A_{h}$, and a constant $c \equiv c(n, N, p)$ such that $A_{h} \rightarrow A$ uniformly on compact sets,

$$
\begin{gathered}
\left|A_{h}(z)\right| \leq c L\left(\mu^{2}+\frac{1}{h^{2}}+|z|^{2}\right)^{\frac{p-1}{2}} \\
\frac{\partial A_{h, 1}^{i}}{\partial z_{\beta}^{j}}(z) \xi_{i}^{\alpha} \xi_{j}^{\beta} \geq c^{-1} \nu\left(\mu^{2}+\frac{1}{h^{2}}+|z|^{2}\right)^{\frac{p-2}{2}}|\xi|^{2} \\
\left|\frac{\partial A_{h, \alpha}^{i}}{\partial z_{\beta}^{j}}(z)\right| \leq \Lambda\left(\mu^{2}+\frac{1}{h^{2}}+|z|^{2}\right)^{\frac{p-2}{2}}
\end{gathered}
$$

Proof. Let us set

$$
B^{i}(z)=\left(\mu^{2}+|z|^{2}\right)^{\frac{p-2}{2}} z_{\alpha}^{i} \quad i=1, \ldots . N ; \alpha=1, \ldots, n
$$

with

$$
\left.B(z) \equiv\left(B^{i}(z)\right)\right)
$$

and define for cvery $h \in N$

$$
W_{h}(z)=\left(1-\eta_{h}(z)\right) A(z)+\eta_{h}(z) B(z)
$$

where $\eta \in C^{2}\left(R^{n N}\right)$ is such that $0 \leq \eta_{h} \leq 1$ and

$$
\eta_{h}= \begin{cases}0 & \text { if }|z| \leq h \\ 1 & \text { if }|z| \geq 2 h\end{cases}
$$

It is obvious that

$$
\left|W_{h}(z)\right| \leq c L\left(\mu^{2}+\frac{1}{h^{2}}+|z|^{2}\right)^{\frac{p-1}{2}},
$$

recall that the vector field $B$ is monotone in the sense that: 


$$
\left\langle B_{h}\left(z_{1}\right)-B_{h}\left(z_{2}\right), z_{1}-z_{2}\right\rangle \geq c^{-1} \nu\left(\mu^{2}+\left|z_{1}\right|^{2}+\left|z_{2}\right|^{2}\right)^{\frac{p-2}{2}}\left|z_{1}-z_{2}\right|^{2}
$$

so, by the fact that it is the sum of two monotone vector fields, also $W_{h}$ satisfies:

$$
\left\langle W_{h}\left(z_{1}\right)-W_{h}\left(z_{2}\right), z_{1}-z_{2}\right\rangle \geq c^{-1} \nu\left(\mu^{2}+\left|z_{1}\right|^{2}+\left|z_{2}\right|^{2}\right)^{\frac{p-2}{2}}\left|z_{1}-z_{2}\right|^{2}
$$

for every $z_{1}, z_{2} \in R^{n N}$. Now we denote by $\rho(z)$ a positive radially symmetric mollifier with compact support in $B(0,1)$ and define

$$
A_{h}(z)=\int_{B(0,1)} W_{h}\left(z+\frac{\xi}{h}\right) \rho(\xi) d \xi .
$$

We can finally verify that $A_{h}$ satisfies the required conditions. By definition of $A_{h}$ and (23) we get

$$
\begin{aligned}
\left|A^{h}(z)\right| & \leq c L \int_{B(0,1)} \rho(\xi)\left|\mu^{2}+\frac{1}{h^{2}}+\right| z+\left.\left.\frac{\xi}{h}\right|^{2}\right|^{\frac{p-1}{2}} d \xi \\
& \leq c L\left(\mu^{2}+\frac{1}{h^{2}}+|z|^{2}\right)^{\frac{p-1}{2}}
\end{aligned}
$$

then (20) is verified. Now from (24) it follows, setting

$$
\begin{aligned}
& S=B(0,1) \cap\left\{\xi \in R^{n N}:\langle\xi, z\rangle \geq 0\right\} \\
& \left\langle A_{h}\left(z_{1}\right)-A_{h}\left(z_{2}\right), z_{1}-z_{1}\right\rangle \\
& =\int_{B(0,1)} \rho(\xi)\left\langle W_{h}\left(z_{1}+\frac{\xi}{h}\right)-W_{h}\left(z_{2}+\frac{\xi}{h}\right) ;\left(z_{1}\right)-\left(z_{2}\right)\right\rangle d \xi \\
& \geq c^{-1} \nu \int_{B(0,1)} \rho(\xi)\left(\mu^{2}+\left|z_{1}+\frac{\xi}{h}\right|^{2}+\left|z_{2}+\frac{\xi}{h}\right|^{2}\right)^{\frac{p-2}{2}} d \xi\left|z_{1}-z_{2}\right|^{2} \\
& \left.\geq c^{-1} \nu \int_{S} \rho(\xi)\left(\mu^{2}+\frac{|\xi|^{2}}{h^{2}} \pm\right\rfloor-\left.z_{L}\right|^{2}+\left.1 z_{2}\right|^{2}+2 \frac{\left\langle\xi, z_{1}\right\rangle}{h}+2 \frac{\left\langle\xi, z_{2}\right\rangle}{h}\right)^{\frac{q^{-2}}{2}} d \xi \times \\
& \times\left|z_{1}-z_{2}\right|^{2} \\
& \geq c^{-1} \nu\left(\mu^{2}+\frac{1}{h^{2}}+\left|z_{1}\right|^{2}+\left|z_{2}\right|^{2}\right)^{\frac{n-2}{2}}\left|z_{1}-z_{2}\right|^{2},
\end{aligned}
$$

and this implies (21) since $A_{h}$ is a $C^{1}$ function. To conclude the proof we observe that $W_{h}(z) \equiv A(z)$ if $|z| \leq h ;$ while $W_{h}(z) \equiv B(z)$ if $|z| \geq 2 h$; 
then arguing as before we deduce that $A_{h}$ satisfies (22) and $A_{h} \rightarrow A$ uniformly on compact sets.

In the sequel $V(z)$ will denote the following function

$$
V(z)=\left(\mu^{2}+|z|^{2}\right)^{\frac{p-2}{4}} z,
$$

where $z \in R^{n N}, \mu \geq 0, p>1$.

Next lemma will be useful in the next section and its proof can be found in $[\mathrm{AF}]$

Lemma 3. The function $V(z)$ satisfies:

$$
c_{o}^{-1}\left|z_{1}-z_{2}\right|^{2} \leq \frac{\left|V\left(z_{1}\right)-V\left(z_{2}\right)\right|^{2}}{\left(\mu^{2}+\left|z_{1}\right|^{2}+\left|z_{2}\right|^{2}\right)^{\frac{p-2}{2}}} \leq c_{o}\left|z_{1}-z_{2}\right|^{2}
$$

for any $z_{1}, z_{2} \in R^{n N}$, where $c_{o}$ depends only on $N, n, p$, and $\mu \geq 0, p>1$.

\section{Proof of the regularity results}

In this section we prove Proposition 1 and Proposition 2, assuming that $A(z)$ only satisfies (7) and (8), thus obtaining the existence of second derivatives for solutions of (1) under fairly general assumption on $A$. In the scalar case, we are also able to prove local boundedness of the gradient of solutions of (1).

Let us first consider the

\section{Scalar case.}

Theorem 1. Let $A: R^{n} \rightarrow R^{n}$ be a continuous function satisfying (7) and (8) with $\mu \geq 0$, and $u \in W^{1, p}(\Omega)$ a local solution of (1). Then

$$
V(D u) \in W_{l o c}^{1,2}\left(\Omega, R^{n}\right), \quad D u \in L_{l o c}^{\infty}\left(\Omega, R^{n}\right)
$$

and for any ball $B_{R}\left(x_{o}\right) \subset \Omega, 0<\rho<R$

$$
\begin{gathered}
\int_{B_{\rho}}|D V(D u)|^{2} d x \leq \frac{C}{(R-\rho)^{2}} \int_{B_{R}}\left(\mu^{2}+|D u|^{2}\right)^{\frac{p}{2}} d x \\
\sup _{B_{\rho}}|D u|^{p} \leq c(\rho) f_{B_{R}}\left(\mu^{2}+|D u|^{2}\right)^{\frac{p}{2}} d x .
\end{gathered}
$$


Before proving the theorem we remark that estimate above inplies that $u \in W_{l o c}^{2,2}(\Omega)$ if $\mu>0$.

Corollary 1. Under the assumptions of theorem 1, if $\mu>0$, then $u \in W_{l \infty c}^{2,2}(\Omega)$ and for every ball $B_{R}\left(x_{o}\right) \subset \Omega, 0<\rho<R$

$$
\int_{B_{\rho}}\left(\mu^{2}+|D u|^{2}\right)^{\frac{p-2}{2}}\left|D^{2} u\right|^{2} d x \leq \frac{C}{(R-\rho)^{2}} \int_{B_{R}}\left(\mu^{2}+|D u|^{2}\right)^{\frac{p}{2}} d x
$$

Proof. Let us fix $s \in\{1, \ldots, n\}$ and $0<\rho<R$. If $|h| \leq \frac{R-\rho}{2}$ we get from (25) and (26)

$$
\begin{aligned}
& \int_{B_{\rho}}\left(\mu^{2}+|D u(x)|^{2}+\left|D u\left(x+h e_{s}\right)\right|^{2}\right)^{\frac{p-2}{2}}\left|\Delta_{h, s}(D u)\right|^{2} d x \\
& \leq c \int_{B_{\rho}}\left|\Delta_{h, s}(\bigvee(D u))\right|^{2} d x \leq \int_{B_{\frac{R+\rho}{2}}}|D V(D u)|^{2} d x \\
& \leq \frac{c}{(R-\rho)} \int_{B_{R}}\left(\mu^{2}+|D u|^{2}\right)^{\frac{p}{2}} d x
\end{aligned}
$$

The result then follows, recalling that $D u$ is locally bounded, letting $h \rightarrow 0$.

\section{Proof of Theorem 1.}

Let us fix $B_{R}\left(x_{o}\right) \subset \Omega$ and denote by $u_{h}$ the solution of the equation

$$
\int_{B_{R}} A_{h, \alpha}^{i}\left(D u_{h}\right) D_{\alpha} \varphi d x=0 \quad \forall \varphi \in W_{o}^{1, p}\left(B_{R}\right)
$$

where $u_{h} \in u+W_{o}^{1, p}\left(B_{R}\right)$, and $A_{h}$ is the sequence given by lemma 2; ihen we have

$$
\begin{gathered}
\int_{B_{R}}\left\langle A_{h}\left(D u_{h}\right)-A_{h}(D u), D u-D u_{h}\right\rangle d x= \\
\int_{B_{R}}\left\langle A(D u)-A_{h}(D u), D u-D u_{h}\right\rangle d x
\end{gathered}
$$


Using assumption (7); and inequalities (20), (21) we have

$$
\begin{aligned}
& c^{-1} \int_{B_{R}}\left(\mu^{2}+\frac{1}{h^{2}}+\left|D u_{h}\right|^{2}+|D u|^{2}\right)^{\frac{p-2}{2}}\left|D u-D u_{h}\right|^{2} d x \leq \\
& \leq c L \int_{B_{R}}\left(\mu^{2}+\frac{1}{h^{2}}+|D u|^{2}\right)^{\frac{p-1}{2}}\left|D u-D u_{h}\right| d x
\end{aligned}
$$

From this inequality we can deduce

$$
\int_{B_{R}}\left|D u-D u_{h}\right|^{p} d x \leq C \int_{B_{R}}\left(\mu^{2}+\frac{1}{h^{2}}+|D u|^{2}\right)^{\frac{p-1}{2}}\left|D u-D u_{h}\right| d x \text {. }
$$

This is obvious for $p \geq 2$. Otherwise, if $1<p<2$ notice that

$$
\begin{aligned}
& \left|D u-D u_{h}\right|^{p} 1_{\left\{x \in B_{R}:\left|D u_{h}(x)\right|^{2} \geq 4|D u(x)|^{2}+\mu^{2}+\frac{1}{h^{2}}\right\}} \\
& \leq c\left[\left(\mu^{2}+\frac{1}{h^{2}}\right)+\left|D u_{h}\right|^{2}+|D u|^{2}\right]^{\frac{p-2}{2}}\left|D u-D u_{h}\right|^{2} \\
& \leq\left(\mu^{2}+\frac{1}{h^{2}}+\left|D u_{h}\right|^{2}+|D u|^{2}\right)^{\frac{p-2}{2}}\left|D u-D u_{h}\right|^{2} .
\end{aligned}
$$

From (28) we obtain applying Young inequality

$$
\int_{B_{R}}\left|D u-D u_{h}\right|^{p} d x \leq c \int_{B_{R}}\left(\mu^{2}+\frac{1}{h^{2}}+|D u|^{2}\right)^{\frac{p}{2}} d x
$$

and this implies that

$$
\int_{B_{R}}\left|D u_{h}\right|^{p} d x \leq C
$$

From this inequality and Proposition 1 we have that for any $0<\rho<R$

$$
\int_{B_{\rho}}\left|D V_{h}\left(D u_{h}\right)\right|^{2} d x \leq c(\rho)
$$

where

$$
V_{h}(z)=\left(\mu^{2}+\frac{1}{h^{2}}+|z|^{2}\right)^{\frac{p-2}{4}} z,
$$

and $c(\rho)$ is independent on $h$. Eventually passing to a (not relabelled) subsequence, we may assume that:

$$
\begin{cases}u_{h} \rightarrow \bar{u} & \text { weakly in } W^{1, p}\left(B_{R}\right) \\ V_{h}\left(D u_{h}\right) \rightarrow g & \text { weakly in } W_{\text {loc }}^{1,2}\left(B_{R}\right) \\ V_{h}\left(D u_{h}\right) \rightarrow g & \text { in } L_{\text {loc }}^{2}\left(B_{R}\right) \text { and a.e. in } B_{R}\end{cases}
$$


Now we observe that $(31)_{3}$ implies that $\left|D u_{h}\right|$ 'converges a.e. and therefore that $D u_{h}$ converges a.e. in $B_{R}$. Arguing as in the proof of (28) we also have for any $h$

$$
\left|D u_{h}\right|^{p} 1_{\left\{x \in B_{R}:\left.D u_{h}(x)\right|^{2} \geq \mu^{2}+\frac{1}{h^{2}}\right\}} \leq c\left|V_{h}\left(D u_{h}\right)\right|^{2},
$$

and we may assume that

$$
D u_{h} \rightarrow D \bar{u} \text { in } L_{l o c}^{p}\left(B_{R}\right) \text { and a.e. in } B_{R} .
$$

Using (32) we can pass to the limit as $h \rightarrow \infty$ in the equation

$$
\int_{B_{R}}\left\langle A_{h}\left(D u_{h}\right), D \varphi\right\rangle d x=0 \quad \forall \varphi \in W_{o}^{1, p}\left(B_{R}\right)
$$

to get that

$$
\int_{B_{R}}\langle A(D \bar{u}), D \varphi\rangle d x=0 \quad \forall \varphi \in W_{o}^{1, p}\left(B_{R}\right)
$$

moreover we have

$$
V_{h}\left(D u_{h}\right) \rightarrow V(D \bar{u}) \quad \text { weakly in } W_{l o c}^{1,2}\left(B_{R}\right) .
$$

We now recall that assumption (8) implies the uniqueness of the solution of (1) and then from (33) we deduce that $u=\bar{u}$. Finally from Proposition 2 we have

$$
\begin{gathered}
\int_{B_{\rho}}\left|D V\left(D u_{h}\right)\right|^{2} d x \leq \frac{C}{(R-\rho)^{2}} \int_{B_{R}}\left(\mu^{2}+\frac{1}{h^{2}}\left|D u_{h}\right|^{2}\right)^{\frac{p}{2}} d x \\
\operatorname{sip}_{B_{\rho}}\left|D u_{h}\right|^{p} \leq c(\rho) f_{B_{R}}\left(\mu^{2}+\left|D u_{h}\right|^{2}\right)^{\frac{p}{2}} d x,
\end{gathered}
$$

and from this inequalities, passing to the limit as $h \rightarrow 0$ we get the result.

\section{The vectorial case.}

In this case we cannot get the local boundedness of $D u$ and we may only prove the existence of second derivatives. 
Theorem 2. Let $A: R^{n N} \rightarrow R^{n N}$ be a continuous function satisfying (2) and (3) with $\mu \geq 0$, and $u \in W^{1, p}(\Omega)$ a local solution of (1). Then $V(D u) \in W_{l o c}^{1,2}\left(\Omega, R^{n N}\right)$ and for any ball $B_{R}\left(x_{o}\right) \subset \Omega, 0<\rho<R$

$$
\int_{B_{\rho}}|D V(D u)|^{2} d x \leq \frac{C}{(R-\rho)^{2}} \int_{B_{R}}\left(\mu^{2}+|D u|^{2}\right)^{\frac{p}{2}} d x .
$$

Moreover, if $\mu>0 u \in W_{l o c}^{2,2}\left(\Omega, R^{N}\right)$ when $p \geq 2$, otherwise $u \in$ $W_{\text {loc }}^{2, p}\left(\Omega, R^{N}\right)$ when $1<p<2$ and the following estimate holds

$$
\int_{B_{\rho}}\left(\mu^{2}+|D u|^{2}\right)^{\frac{p-2}{2}}\left|D^{2} u\right|^{2} d x \leq \frac{C}{(R-\rho)^{2}} \int_{B_{R}}\left(\mu^{2}+|D u|^{2}\right)^{\frac{p}{2}} d x
$$

Proof. The first part of the theorem can be proved in the same way as in the scalar case. To prove the second part of the theorem one may argue as in the proof of corollary 5 to get

$$
\begin{aligned}
& \int_{B_{\rho}}\left(\mu^{2}+|D u(x)|^{2}+\left|D u\left(x+h e_{s}\right)\right|^{2}\right)^{\frac{p-2}{2}}\left|\Delta_{h, s}(D u)\right|^{2} d x \\
& \leq c \int_{B_{\rho}}\left|\Delta_{h, s}(V(D u))\right|^{2} \leq \int_{B_{(R+\rho) / 2}}|D V(D u)|^{2} d x \\
& \leq \frac{C}{(R-\rho)^{p}} \int_{B_{R}}\left(\mu^{2}+|D u|^{2}\right)^{\frac{p}{2}} d x,
\end{aligned}
$$

for any $B_{R} \subset, 0<\rho<R, s \in\{1, \ldots, n\}$. From this estimate, for $p \geq 2$, letting $h$ go to 0 we have(35) while for $1<p<2$ we deduce

$$
\begin{aligned}
& \int_{B_{\rho}}\left|\triangle_{h}(D u)\right|^{p} d x \\
& \leq\left[\int_{B_{\rho}}\left(\mu^{2}+|D u(x)|^{2}+\left|D u\left(x+h e_{s}\right)\right|^{2}\right)^{\frac{p-2}{2}}\left|\Delta_{h, s}(D u)\right|^{2} d x\right]^{\frac{p}{2}} . \\
& \cdot\left[\int_{B_{\rho}}\left(\mu^{2}+|D u(x)|^{2}+\left|D u\left(x+h e_{s}\right)\right|^{2}\right)^{\frac{p}{2}} d x\right]^{\frac{2-p}{2}} \\
& \leq \frac{C}{(R-\rho)^{p}} \int_{B_{R}}\left(\mu^{2}+|D u|^{2}\right)^{\frac{p}{2}} d x,
\end{aligned}
$$

and the result again follows letting $h \rightarrow 0$. 


\section{References}

[AF] Acerbi E. and N. Fusco, Regularity for minimizers of non-quadratic functionals: the case $1<p<2$, J. Math.Anal.Appl. 140 (1989), 115-135.

[C] Campanato S., Hölder continuity of the solutions of some nonlinear elliptic systems, Adv. in Math. 48 (1989), 16-49

[DB] Di Benedetto E., $C^{1, \alpha}$ local regularity of weak solutions of degenerate elliptic equations, Nonlinear Anal.7 (1983), 827-850.

[E] Esposito L., A remark on $C^{1, \alpha}$ regularity of minimizers, to appear in Ric. di Mat.

[EFL] Esposito L., Fusco N., Leonetti F., A remark on the existence of second derivatives of minimizers of convex integrals. Preprint.

[EG] Evans L.C. and R.F. Gariepy, Measure theory and fine properties of functions, Studies in Advanced Math., CRC Press (1999).

[FF] Fonseca I. and N. Fusco, Regularity results for anisotropic image segmentation models, to appear in Ann. Scuola Norm. Sup.

[G] Giaquinta M., Multiple integrals in calculus of variations and nonlinear elliptic sistems, Annals of Math. Studies 105, Princeton Univ. Press, (1983)

[GM] Giaquinta M. and G. Modica, Regularity results for some classes of higher order nonlinear elliptic systems, J.Reine Ang.Math., 311/312 (1979), 145-169.

[Gi] Giusti E., Metodi diretti nel Calcolo delle Variazioni, U.M.I 1994

[LU] Ladyzenskaya $\mathrm{O}$. and N. Ural'ceva, Linear and quasilinear elliptic equations, Academic Press, New York, (1968) (second russian edition, Nauka (1973))

[T] Tolksdorf P., Everywhere regularity for some quasilinear systems with lack of ellipticity Ann. Mat. Pura e Appl. 194 (1989), 214-266

[U] Uhlenbeck K., Regularity for a class of nonlinear elliptic systems, Acta Math. 138(1977), 219-240. 
Dipartimento di Matematica e Applicazioni "R. Caccioppoli", Università di Napoli "Federico II",

Complesso Monte S. Angelo

Via Cintia

80126 Napoli

Italia

Recibido: 3 de Julio de 1996

Revisado: 4 de Noviembre de 1996 